\title{
Evolução de propriedades físicas do solo em função dos sistemas de manejo em culturas anuais
}

\section{Evolution of physical properties of soils according to tillage systems on annual crops}

\author{
Rafael Fuentes Llanillo ${ }^{1}$; Alfredo Richart ${ }^{1} ;$ João Tavares Filho $^{2 *}$; \\ Maria de Fátima Guimarães ${ }^{2}$; Rogério R. M. Ferreira ${ }^{1}$
}

\begin{abstract}
Resumo
Para assegurar a sustentabilidade do sistema produtivo, o manejo do solo deve manter as propriedades físicas do solo o mais próximo das condições originais em que este se encontrava na natureza. Esta revisão sintetiza os efeitos de três sistemas de manejo de solo (convencional, mínimo e direto) em culturas anuais sobre as propriedades físicas do solo como densidade, porosidade, resistência à penetração, velocidade de infiltração, condutividade hidráulica, compressibilidade, nível de matéria orgânica, tamanho e estabilidade de agregados. O plantio direto apresenta vantagens nos níveis de matéria orgânica, na estabilidade de agregados, na compressibilidade e na condutividade hidráulica, mas tem suas limitações quanto à densidade do solo e resistência à penetração. O preparo mínimo à base de escarificação apresenta-se especialmente eficiente na velocidade de infiltração, na condutividade hidráulica e na resistência à penetração, sendo intermediário entre convencional e direto nos demais aspectos. $\mathrm{O}$ preparo convencional, principalmente em condições tropicais, apresenta os indicadores menos favoráveis para propriedades físicas do solo, normalmente mais distante das condições da cobertura vegetal original e, em certas situações, aproximando-se do preparo mínimo e do plantio direto. Nesse particular, os sistemas conservacionistas se aproximam em alguns aspectos das condições naturais, mas não reproduzem as condições das coberturas naturais originais de mata, cerrado ou campos nativos, mesmo que estando na direção da sustentabilidade.
\end{abstract}

Palavras-chave: Densidade do solo, matéria orgânica, estabilidade de agregados, compactação, plantio direto

\begin{abstract}
Soil management must keep the soil physical properties next to the original conditions in natural systems to assure the sustainability of agricultural systems. This review synthesizes the effects of conventional tillage, minimum tillage and no-tillage systems of annual crops, on soil physical properties as bulk density, porosity, soil resistance to root penetration, infiltration speed, hydraulic conductivity, compressibility, organic matter level, soil aggregate size and stability. No-tillage presents advantages on organic matter level, size and stability of aggregates, compressibility and hydraulic conductivity but has
\end{abstract}

1 Doutorando de Agronomia da Universidade Estadual de Londrina.

2 Professor do Departamento de Agronomia da Universidade Estadual de Londrina, Caixa Postal 6001, 86051-990, Londrina, PR. E-mail: tavares@uel.br.

* Autor para correspondência. 
limitations on bulk density and resistance to root penetration. Minimum tillage with chisel plow is specially efficient in relation to infiltration speed and hydraulic conductivity, and intermediate between conventional and no-tillage in other aspects. Conventional tillage with total pulverization of soil surface, mainly on tropical conditions, presents the less favorable scores on soil physical properties, close to minimum tillage and no-till only in few circumstances, and frequently the most different from the natural conditions. The conservation systems by their side, despite of similarities in some aspects with natural conditions, are not able to reproduce the conditions of natural forests, savannas or natural pastures, but are in the sustainability direction.

Key words: Soil bulk density, organic matter, aggregate stability, compaction, no-tillage

\section{Introdução}

A ampla adoção de tecnologias de produção mecanizada de grãos no Brasil a partir do início dos anos 1970 impulsionou a degradação dos solos pela pulverização superficial, pela diminuição da matéria orgânica, pela compactação e pelo subseqüente processo erosivo que afetou a sustentabilidade dos sistemas, principalmente pelo declínio da produtividade. Como resposta a esse processo de deterioração, novos sistemas com orientação conservacionista como o cultivo mínimo e o plantio direto vêm sendo desenvolvidos para as condições subtropicais visando diminuir o revolvimento do solo, principalmente no preparo primário. Pesquisas sobre sistemas conservacionistas iniciaram-se a partir da segunda metade da década de 1970, com maior ênfase nas décadas de 1980 e 1990.

De acordo com Denardin e Kochhann (1997), as mobilizações intensivas do solo, principalmente no sistema convencional, sob condições inadequadas de umidade e de cobertura vegetal, modificam adversamente a estrutura do solo, afetando basicamente as relações entre as fases sólida, líquida e gasosa. Isto subdivide a camada arável em uma parte que é superficial e pulverizada, e outra que é subsuperficial e compactada. Por outro lado, no sistema de plantio direto (PD) a semeadura é feita sem preparo do solo, mantendo os restos culturais na superfície. Esse sistema de manejo é eficaz na proteção da superfície do solo contra agentes erosivos. Mas como não há revolvimento do solo, pode ocorrer formação de camadas compactadas pela distribuição das pressões exercidas na superfície do solo pelas máquinas/implementos ou casco de animais, em áreas de integração lavoura-pecuária (CRUZ et al., 2003). O sistema convencional de preparo do solo tem ocasionado compactação subsuperficial em função da descompactação mecânica da camada mobilizada, sendo que a carga aplicada tem efeito cumulativo em subsuperfície ao longo do tempo; e no sistema PD, a compactação é mais superficial pela não-mobilização do solo e concentração das cargas cumulativas na camada superficial dos solos ao longo do tempo (HAKANSSON; VOORHEES; RILEY, 1988; TORMENA; SILVA; LIBARDI, 1998). Essa compactação é considerada o principal aspecto negativo do sistema de PD e tem motivado alguns agricultores, ainda que temporariamente, a retornar ao preparo convencional do solo, visando corrigir essa limitação (TORMENA; ROLOFF, 1996). Mesmo que sem retornar ao preparo convencional, escarificações eventuais têm sido um instrumento utilizado pelos produtores, a despeito da discordância de boa parte do setor técnico.

A principal premissa para avaliar a sustentabilidade de um sistema de manejo é que ele permita manter as propriedades físicas do solo o mais próximo das condições originais em que este se encontrava na natureza, na maior parte das vezes sob cobertura de matas, mas também sob outros tipos de cobertura vegetal. Como contraponto, mais que a proximidade das condições naturais, está a capacidade do solo continuar produzindo indefinidamente de forma econômica. 


\section{Indicadores de qualidade física dos solos e efeitos dos sistemas de manejo nas propriedades físicas}

Com relação a indicadores de qualidade física dos solos, Singer e Ewing (2000) e Imhoff (2002) sugerem que a escolha deve recair sobre aqueles que estão relacionados diretamente à produção das culturas e que sejam suficientemente potentes para medir a capacidade do solo de fornecer adequada aeração e quantidade de água para o crescimento e expansão do sistema radicular, da mesma forma que devem medir a magnitude com a qual a matriz do solo resiste à deformação. Os indicadores mais utilizados de qualidade física do solo são a densidade, a porosidade total, a distribuição e o tamanho dos poros, a distribuição das partículas, a resistência do solo à penetração, a profundidade efetiva de enraizamento, o intervalo hídrico ótimo, o índice de compressão, o diâmetro médio de agregados e o nível de matéria orgânica (TOPP et al., 1997; SCHOENHOLTZ; VAN MIEGROET; BURGER, 2000; SINGER; EWING, 2000). Com base em alguns trabalhos clássicos são apresentadas algumas contribuições mais recentes sobre os impactos dos diferentes sistemas de manejo nas propriedades físicas do solo.

\section{Densidade}

A propriedade física mais estudada e monitorada é a densidade do solo. A variação nos valores da densidade do solo, em sua maior parte, é proveniente das diferenças no volume total de poros, de modo que densidade e porosidade estão muito relacionadas e por isso são apresentadas de forma conjunta. Geralmente, solos cultivados com preparo convencional perdem matéria orgânica e sofrem aumento de densidade (ALLMARAS; BLACK; RICKMAN, 1973) abaixo da camada de corte dos implementos de preparo. Já no sistema de PD têm sido constatada uma compactação na camada mais superficial até $10 \mathrm{~cm}$, principalmente em solos argilosos, devido ao tráfego de máquinas e implementos como evidenciaram Vieira, Cogo e
Cassol (1978), Abrão et al. (1979), Fernandes, Galloway e Bronson (1983), Eltz, Peixoto e Jaster (1989) e Derpsch et al. (1991). Entretanto, Machado e Brum (1978) encontraram resultado diferente, com o PD apresentando menor densidade que no preparo convencional, na camada superficial.

Derpsch et al. (1991) em Latossolo Roxo distrófico argiloso no Norte do Paraná em área experimental sem tráfego de colheita, após sete anos de preparos diferenciados de solo, verificaram que em PD a densidade entre 0 e $20 \mathrm{~cm}$ foi maior, nesse caso da ordem de $1,17 \mathrm{Kg} \mathrm{dm}^{-3}$ contra $1,10 \mathrm{Kg} \mathrm{dm}^{-3}$ do preparo mínimo e $1,02 \mathrm{Kg} \mathrm{dm}^{-3}$ do convencional. Entre 20 a $30 \mathrm{~cm}$ ocorreu o inverso, apresentando o preparo convencional na camada chamada de "péde-arado" maiores densidades, atingindo $1,10 \mathrm{Kg} \mathrm{dm}^{-3}$ contra $1,03 \mathrm{Kg} \mathrm{dm}^{-3}$ do PD. Diferentemente, Eltz, Peixoto e Jaster (1989) após sete anos de comparação entre cinco sistemas de manejo (PC/PC), PC/PD, $\mathrm{PD} / \mathrm{PC}, \mathrm{ESC}$ (escarificação)/PD e $\mathrm{PD} / \mathrm{PD}$ ) em Latossolo Bruno álico em altitude superior a $1100 \mathrm{~m}$, em Guarapuava (PR), não encontraram diferenças significativas de densidade, nem entre manejos nem ao longo do perfil, que se mantiveram próximas de $1,00 \mathrm{Kg} \mathrm{dm}^{-3}$ na profundidades $0-40 \mathrm{~cm}$.

Em geral a porosidade, da mesma forma que a densidade, é bastante alterada pelo sistema de preparo de solo. Para Eltz, Peixoto e Jaster (1989), diferentes implementos e condições de umidade do solo no preparo, tipos de solo e de clima acarretam resultados diferentes de porosidade. Dalla Rosa (1981), trabalhando com Latossolo Roxo, observou a presença de camada compactada abaixo da camada mobilizada pelo arado, onde a macroporosidade e a infiltração de água foram drasticamente diminuídas. Vieira (1981), Fernandes, Galloway e Bronson (1983) e Derpsch et al. (1991) observaram diminuição de macroporos em PD comparado ao preparo convencional em um Latossolo Roxo. Diferentemente, Eltz, Peixoto e Jaster (1989) após sete anos para o Latossolo Bruno, não encontraram diferença entre sistemas de manejo para porosidade total, macroporos e microporos, o que foi atribuído à 
altitude, ao clima mais frio e ao alto conteúdo natural de matéria orgânica na superfície desse solo.

Em Latossolo Roxo em Jaboticabal (SP), Corsini e Ferraudo (1999) encontraram densidades de 1,15 $\mathrm{Mg} \mathrm{m}^{-3}$ a $15 \mathrm{~cm}$ atingindo, após oito anos de manejo diferenciado, 1,16 $\mathrm{Mg} \mathrm{m}^{-3} \mathrm{em}$ PD e 1,25 $\mathrm{Mg} \mathrm{m}^{-3} \mathrm{em}$ PC. Os macroporos que eram de $24 \%$ no início do experimento, foram para $23 \%$ em PD e $13 \%$ em PC oito anos depois. Os autores concluíram que nos três primeiros anos agrícolas, o sistema de PD diminui a porosidade e o potencial de desenvolvimento radicular da camada superficial do solo; somente a partir do quinto ano agrícola é que esses parâmetros começam a crescer.

Em Latossolo Vermelho distrófico de textura média do Arenito Caiuá, em Araruna (PR), após dois anos sob cultivo de mandioca em preparo convencional, preparo mínimo e PD, Tormena et al. (2002) encontraram na camada $0-10 \mathrm{~cm}$ as densidades de $1,67 \mathrm{Mg} \mathrm{m}^{-3}$ no PD, 1,55 $\mathrm{Mg} \mathrm{m}^{-3}$ no preparo mínimo e $1.45 \mathrm{Mg} \mathrm{m}^{-3}$ no PC. Os preparos convencional e mínimo promoveram aumentos de macroporosidade e porosidade total pelo uso do arado e do escarificador. A microporosidade foi maior no PD. O aumento da densidade do solo no PD ocorreu às expensas dos poros de maior diâmetro. Araújo, Tormena e Silva (2004a), nesse mesmo solo em Maringá (PR), compararam uma área sob mata com outra cultivada em PC durante vinte anos, chegando a densidades, na camada $0-20 \mathrm{~cm}$, de $1,48 \mathrm{Mg} \mathrm{m}^{-3}$ na mata contra $1,71 \mathrm{Mg} \mathrm{m}^{-3}$ do sistema convencional, porosidade total de $41 \%$ na mata contra $30 \%$ no convencional e macroporos com $16 \%$ na mata contra $6 \%$ no convencional.

A porosidade total é geralmente calculada a partir das medidas de densidade (KAY; VANDENBYGAART, 2002). Em países temperados, constata-se que, em geral, em comparações de sistemas com menos de dez anos, a densidade é maior e a porosidade é menor em PD do que em PC na camada $0-10 \mathrm{~cm}$ (DOUGLAS et al., 1986; HUSSEIN; OLSEN; SIEMENS, 1998;
VANDENBYGAART; PROTZ; TOMLIN, 1999; SCHJONNING; RASMUSSEN, 2000). Registrouse também uma referência de maior densidade do solo em PD que convencional após a fase de pousio, mas com densidades semelhantes depois de cevada em rotação (FRANZLUEBBERS; ARSHAD, 1996) e outra com densidades semelhantes em seis solos do Canadá (ANGERS et al., 1997) e apenas uma referência de maior densidade do solo em convencional que em PD (EKEBERG; RILEY, 1997).

Diferenças na densidade do solo em comparações de manejos com mais de 15 anos são mais consistentes, pois registram os efeitos cumulativos na porosidade total, compactação, desenvolvimento de pé-de-arado, desenvolvimento de fauna do solo e distribuição da matéria orgânica. Yang e Kay (2001) observaram, após dezenove anos no Canadá, em solos de texturas argilosa e arenosa, que a densidade foi maior em PD que em convencional nos $20 \mathrm{~cm}$ superiores, sendo a maior diferença a $5-10 \mathrm{~cm}$ com $1,58 \mathrm{Mg} \mathrm{m}^{-3}$ contra $1,40 \mathrm{Mg} \mathrm{m}^{-3}$, respectivamente. A menor densidade nos dois sistemas foi encontrada na camada $0-5 \mathrm{~cm}$ e o teor de matéria orgânica foi maior em PD que no PC, o que, provavelmente, diminuiu a diferença de densidade e porosidade; não houve evidência de "pé-de-arado". Outros trabalhos apontam essa mesma tendência, como Tebrügge e Düring (1999) em cinco locais na Alemanha em comparações de dez e 18 anos e Gomez et al. (1999) em Vertissolos no sul da Espanha em comparações de 15 anos.

Alterações na densidade do solo a partir da redução no preparo de solo e uso de escarificação apresentam alterações menos perceptíveis. Yang e Kay (2001) encontraram densidades similares entre PC e preparo com escarificação ao fim de vinte anos num solo siltoso de Ontário, Canadá. Mahboubi, Lal e Faussey (1993) e Lal, Mahuobi e Faussey (1994) não encontraram diferenças significativas na densidade entre escarificação e PD aos sete e 28 anos em Ohio, da mesma maneira que Azooz, Arshad e Franzluebbers (1996) e Franzlubbers e Ashad (1996) aos 13 e 16 anos em British Columbia, Canadá. 
Kay e Vandenbygaart (2002) concluíram que, em zonas temperadas, apesar dos poucos dados sobre solos de textura fina, a introdução do PD resulta na perda de espaços porosos, confirmada pelos aumentos de densidade do solo. Essa perda é mais evidente após 15 anos e é geralmente limitada a camada $5-20 \mathrm{~cm}$. A porosidade nos primeiros $5 \mathrm{~cm}$ pode ser maior no $\mathrm{PD}$, dependendo do aumento da matéria orgânica e da ação da macrofauna.

Avaliações de longo prazo no Brasil apresentam tendências semelhantes. Uma avaliação comparativa de quinze anos entre PD e cinco tipos de PC foi feita por Falleiro et al. (2003) para um Argissolo VermelhoAmarelo câmbico em Coimbra (MG), que encontraram que a densidade do solo foi superior na semeadura direta, comparativamente à dos demais, na média das três profundidades da amostragem (05, 5-10 e 10-20 cm). A maior densidade do solo em sistema de semeadura direta $\left(1,33 \mathrm{Mg} \mathrm{m}^{3}\right.$ na semeadura direta contra 1,16 a $1,20 \mathrm{Mg} \mathrm{m}^{-3}$ nos cinco sistemas convencionais) deveu-se ao acomodamento natural do solo, dada a ausência de revolvimento. Resultados semelhantes, na mesma área, foram obtidos por Sampaio (1987), Siqueira (1989), Siqueira (1995) e Vallejos (1998), assim como De Maria, Castro e Souza Dias (1999), em Oxissolos. Já Klepker e Anghinoni (1995) não encontraram diferenças na densidade do solo, entre seis sistemas de preparo (PD, arado de discos, arado de aivecas, grade pesada, grade pesada mais arado de discos e grade pesada mais arado de aivecas), em um solo arenoso.

Costa et al. (2003) compararam PC, PD e mata nativa aos oito e 21 anos em um Latossolo Bruno alumínico câmbico em altitude superior a $1000 \mathrm{~m}$, em Guarapuava (PR) e observaram que no solo sob $\mathrm{PC}$, houve incremento da densidade do solo entre oito e 21 anos, indicando que a degradação física acentua-se em PC, mesmo com rotação de culturas e manutenção dos resíduos culturais no solo. No solo sob PD, houve aumento na densidade apenas na camada superficial (0-0,05 m) e nas demais camadas houve redução na densidade do solo nesse período. Os autores consideraram que o aumento de densidade em superfície no PD está relacionado ao trânsito de máquinas ou ao adensamento natural, e a diminuição em subsuperfície indica uma melhoria na qualidade física do solo decorrente da atividade da fauna edáfica e de raízes, as quais atuam na formação de canais (bioporos). Estes resultados são semelhantes aos obtidos por Albuquerque, Reinert e Fiorin (1994) e Bertol et al. (2000) e diferem dos apresentados por Abrão et al. (1979), que verificaram maior densidade do solo em subsuperfície sob PD.

Como síntese, pode-se dizer que densidade do solo é um indicador importante, mas insuficiente para explicar a qualidade física do solo. Pelos dados da literatura concluiu-se que, nas condições brasileiras, densidades acima de 1,40 Mg. $\mathrm{m}^{-3}$ para solos argilosos e 1,65 Mg.m ${ }^{-3}$ para solos de textura média são limitantes ao desenvolvimento radicular, assim como $30 \%$ de porosidade total e $10 \%$ de macroporos parecem ser os limites mínimos aceitáveis em relação ao desenvolvimento radicular. Em condições de mata para solos argilosos, as densidades ficam pouco acima de $1,00 \mathrm{Mg} \mathrm{m}^{-3}$ e em solos arenosos ficam acima de $1,40 \mathrm{Mg} \mathrm{m}^{-3}$, em campos nativos do sul do Brasil de 1,00 a $1,20 \mathrm{Mg} \mathrm{m}^{-3}$ e em solos sob cerrado de $0,90 \mathrm{a}$ $1,00 \mathrm{Mg} \mathrm{m}^{-3}$. Solos com altos teores de matéria orgânica na origem pedogenética tem densidades em condições de mata abaixo de $1,00 \mathrm{Mg} \mathrm{m}^{-3}$, e sob cultivo tem mais facilidade para manter a densidade abaixo de $1,10 \mathrm{Mg} \mathrm{m}^{-3}$ mesmo sob PD. Isso ratifica a importância da matéria orgânica no processo de manutenção da qualidade do solo.

\section{Resistência à penetração}

A resistência à penetração (RP) é uma das propriedades físicas que expressa o grau de compactação, e consequentemente, quão facilmente as raízes penetram o solo. Segundo Tormena e Roloff (1996), a penetrometria é um método apropriado para avaliar a resistência à penetração de raízes no solo, apesar das diferenças entre uma raiz e um cone metálico A densidade do solo e a umidade são os fatores mais importantes na quantificação da 
resistência à penetração. A densidade tem uma relação direta com a resistência (BELTRAME et al., 1981; CASTRO, 1995; BORGES et al., 1999) e a umidade está negativamente correlacionada com a resistência (BELTRAME; GONDIM; TAYLOR, 1981; CORRECHEL; SILVA; TORMENA, 1997; TORMENA; SILVA; LIBARDI, 1998).

Estudos relatam que $2 \mathrm{MPa}$ de resistência à penetração traduzem condições impeditivas para o crescimento das raízes e da parte aérea das plantas, o que originalmente foi proposto por Taylor e Gardner (1963). Porém, Azooz, Arshad e Franzluebbers (1996) classificaram como altos valores de resistência à penetração a faixa de 2,0 a 4,0 MPa e Tavares Filho et al. (2001), trabalhando em Latossolo Roxo sob dois sistemas de manejo (PD e convencional) e com a cultura do milho, relatam que valores de resistência do solo à penetração apresentados na literatura como restritivos ao desenvolvimento radicular (1 a 3,5/4,0 $\mathrm{MPa})$, não restringiram o desenvolvimento radicular dessa cultura nos dois sistemas de manejo analisados, porém influenciaram a morfologia do solo.

Tormena e Roloff (1996) estudaram a resistência à penetração num Latossolo Vermelho-Escuro de Ponta Grossa (PR) onde se implantou PD por três anos e monitoraram entrelinhas trafegadas e nãotrafegadas. Na umidade padrão de $0,34 \mathrm{~kg} \mathrm{~kg}^{-1}$ encontraram índices inferiores a 1,6 MPa, que não são impeditivos ao crescimento de raízes. Entretanto, os dados foram concludentes no sentido de detectar que sob tráfego, a resistência era três vezes maior que sem tráfego na faixa de 0 a $10 \mathrm{~cm}$ e que a resistência foi estável nas entrelinhas não trafegadas. Foloni, Calonego e Lima (2003), em Latossolo Vermelho distroférrico de textura média de Botucatu (SP), mediram a resistência à penetração usando camada compactada em anéis de $5 \mathrm{~cm}$ e densidades de $1,28,1,42,1,56$ e $1,69 \mathrm{Mg} \mathrm{m}^{-3}$, concluindo que uma camada compactada com densidade $1,69 \mathrm{Mg}$ $\mathrm{m}^{-3}$ apresenta resistência à penetração de $1,4 \mathrm{MPa}$ impeditiva à penetração de raízes de milho. Tormena et al. (2002), em Latossolo Vermelho distroférrico textura média, cultivado com mandioca em Araruna
(PR), obtiveram os seguintes valores de resistência à penetração na camada $0-5 \mathrm{~cm}: 4,0 \mathrm{MPa} \mathrm{em} \mathrm{PD}$, 2,5 MPa em preparo mínimo e 2,0 MPa em PC, com solo relativamente seco numa umidade em torno de $0,15 \mathrm{~kg} \mathrm{~kg}^{-1}$. Entretanto, com o solo próximo da capacidade de campo encontrou-se 2,7 MPa em PD, 1,8 $\mathrm{MPa}$ no preparo mínimo e 1,4 $\mathrm{MPa}$ em PC, mostrando que na ocorrência de condições mais úmidas, os valores de resistência à penetração podem ser considerados não impeditivos para os sistemas PC e preparo mínimo (PM) e pouco impeditivos para o sistema PD e que, com o secamento do solo, os valores de resistência podem atingir níveis altamente impeditivos, especialmente sob PD (TORMENA; ROLOFF, 1996). Segundo Araújo, Tormena e Silva (2004a) nesse mesmo tipo de solo, em Maringá (PR), sob mata, os valores de resistência do solo à penetração (RP) atingem níveis considerados críticos ao crescimento das raízes $(\mathrm{RP}>2,0 \mathrm{MPa})$ somente com conteúdos de água muito baixos $\left(<0,15 \mathrm{~kg} \mathrm{~kg}^{-1}\right)$ e em densidades elevadas (acima de $1,60 \mathrm{Mg} \mathrm{m}^{-3}$ ). A maior densidade encontrada no solo sob mata nativa $\left(1,59 \mathrm{Mg} \mathrm{m}^{-3}\right)$ é muito próxima da menor densidade encontrada no solo sob cultivo $(1,55 \mathrm{Mg}$ $\left.\mathrm{m}^{-3}\right) \mathrm{e}$, nestes dois pontos, os valores da resistência à penetração, no mesmo conteúdo de água do solo, são muito semelhantes. Solo sob cultivo de vinte anos apresentou resistência à penetração maior que 2,0 MPa abaixo de $0,25 \mathrm{~kg} \mathrm{~kg}^{-1}$ de umidade e acima de $1,65 \mathrm{Mg} \mathrm{m}^{-3}$ de densidade, podendo atingir cerca de 10,0 MPa em condições extremas.

Abreu, Reichert e Reinert (2004), em Argissolo Vermelho Amarelo distrófico arênico de Santa Maria (RS), após sete anos de PD, mediram a RP em quatro sistemas de manejo com umidade de $0,15 \mathrm{~kg} \mathrm{~kg}^{-1}$. A RP foi máxima na camada de $7,5 \mathrm{~cm}$, nos tratamentos sem preparo do solo, atingindo valores máximos de 1,6 MPa no cultivo mínimo (crotalaria), 1,45 MPa no solo descoberto e 1,35 MPa no PD (soja). No sistema escarificação (soja), a RP máxima ocorreu a 17,5 $\mathrm{cm}$, possivelmente na camada não afetada pela mobilização do solo, atingindo $0,85 \mathrm{MPa}$. Em nenhum dos tratamentos a RP superou o limite de 2,0 MPa. 
O confinamento de maiores valores de RP em camadas em torno de $10 \mathrm{~cm}$ de profundidade, com amplitude de 7,5 a $17,5 \mathrm{~cm}$, parece ser uma característica de solos em PD, tanto arenosos (SILVA; REINERT; REICHERT, 2000a; SILVA, 2003; STRECK, 2003) quanto argilosos (GENRO JUNIOR, 2002; SILVA, 2003; SECCO, 2003).

Araújo et al. (2004b), num Latossolo Vermelho distroférrico com $66 \%$ de argila, em Campo Mourão (PR), em área sob PD por 13 anos, testaram o efeito de uma escarificação (ESC) comparada à continuidade do PD e concluíram que a escarificação não surtiu efeito, pois nas camadas $0-15$ e $15-30$ $\mathrm{cm}$ nem as densidades (1,13 e $1,14 \mathrm{Mg} \mathrm{m}^{-3} \mathrm{em}$ PD e 1,09 e $1,14 \mathrm{Mg} \mathrm{m}^{-3}$ em ESC) nem a resistência à penetração, que foi de 2,28 e 2,38 $\mathrm{MPa}$ em PD contínuo e de 2,37 e 2,83 $\mathrm{MPa}$ em PD com escarificação, foram significativamente alteradas. Genro Júnior, Reinert e Reichert (2004), também em Latossolo Vermelho argiloso de Ijuí (RS), em quatro rotações de culturas conduzidas em PD por quatro anos, encontraram valores de resistência à penetração restritivos ao crescimento das plantas (de 3,0 a 3,5 $\mathrm{MPa}$ a $10 \mathrm{~cm}$ ) quando a umidade do solo variou de 0,14 a $0,28 \mathrm{~kg} \mathrm{~kg}^{-1}$. Souza e Alves (2003) em Latossolo Vermelho distrófico de cerrado em Selvíria (MS), compararam quatro sistemas de manejo implantados há oito anos, com um de 16 anos em seringueira e outro de 20 anos em pastagem, e observaram que o cultivo mínimo apresenta resistência do solo à penetração muito parecida com a condição natural sob cerrado, o que deve ser levado em conta na discussão de sustentabilidade. Plantio direto, seringueira e pastagem apresentaram RP próximas de 2,0 MPa de $0-10 \mathrm{~cm}$ e próximo de $3,0 \mathrm{MPa}$ entre $10-20 \mathrm{~cm}$ que podem ser consideradas limitantes.

Costa et al. (2003) para Latossolo Bruno em Guarapuava (PR), após 21 anos de comparação entre $\mathrm{PD}, \mathrm{PC}$ e mata, observaram que a RP variou de 0,5 $\mathrm{MPa}$, no solo sob PC, a 2,0 MPa, no solo sob PD, e não foi afetada significativamente pelos sistemas de manejo devido a um alto coeficiente de variação (42 a 67\%) da resistência à penetração. Apesar disso, o solo sob PD apresentou os maiores valores absolutos de resistência do solo à penetração em todas as camadas $(1,0 \mathrm{MPa}$ a $0-5 \mathrm{~cm}, 1,6 \mathrm{MPa}$ a $5-10 \mathrm{~cm}$ e 2,0 MPa a $10-20 \mathrm{~cm}$ ).

Assim, dependendo das condições de densidade e de umidade, as áreas cultivadas podem rapidamente atingir valores de resistência à penetração maiores que $2 \mathrm{MPa}$, principalmente em $\mathrm{PD}$, mas também em plantio convencional, lavouras permanentes e pastagens. Nas avaliações, apesar de ser uma ferramenta útil, a penetrometria apresenta altos coeficientes de variação, requerendo então um maior número de repetições no campo.

\section{Velocidade de infiltração e condutividade hidráulica}

A dinâmica da água no solo é dependente de suas características intrínsecas (textura, porosidade, profundidade do lençol freático, tipo de argilas, matéria orgânica), de fatores externos (cobertura do solo e densidade de plantas, precipitação, temperatura) e da relação solo-planta-atmosfera. Segundo Abreu, Reichert e Reinert (2004) as três principais características da água que determinam a quantidade e o movimento desta no solo são o potencial matricial, o teor de água e as funções de condutividade hidráulica do solo. O conhecimento das propriedades físico-hídricas do solo é fundamental para entender-se o processo dinâmico dos movimentos de água e de solutos no solo. As variáveis mais usadas para descrever o fluxo de água são a taxa de infiltração, a condutividade hidráulica e o teor e tensão de água no solo (WANG; YATES; ERNST, 1998).

Eltz, Peixoto e Jaster (1989), comparando sucessões em PD (no inverno e verão), preparo mínimo (escarificação no inverno e PD no verão) e três tipos de preparo convencional após sete anos em um Latossolo Bruno de Guarapuava (PR), concluíram que a maior velocidade e volume de água infiltrada ocorreram em preparo mínimo. O PD teve o segundo maior volume infiltrado, devido aos canais formados pelo apodrecimento de raízes e pela ação 
dos organismos do solo mantendo uma velocidade de infiltração mais uniforme durante tempo maior. Derpsch et al. (1991) observaram, em Latossolo Roxo, maiores taxas de infiltração em PD (em torno de $60 \mathrm{~mm} / \mathrm{h}$ ), intermediárias em preparo mínimo, e menores em PC. Alves Sobrinho (2003), trabalhando com um Latossolo Vermelho distroférrico em Dourados (MS), comparando três tratamentos de PD e um convencional, concluíram que o sistema de PD apresentou valores de taxa de infiltração final de água no solo superiores ao preparo convencional e, entre os tratamentos estudados, a sucessão soja - aveia foi a que proporcionou maior valor (superior a $45 \mathrm{~mm} /$ h). Stone e Silveira (1999), estudando sistemas de preparo com arado de aiveca, grade aradora e PD com relação à disponibilidade hídrica da cultura de feijão irrigado num Latossolo Vermelho-Escuro, em Santo Antônio de Goiás (GO), concluíram que houve maior disponibilidade de água em $\mathrm{PD}$, que foi também observada por vários autores (LAL, 1974; VIEIRA, 1981; SIDIRAS; DERPSCH; MONDARDO, 1983; SALTON; MIELNICZUK, 1995). Este fato está relacionado ao não-revolvimento do solo, à menor temperatura e à maior cobertura superficial (VIEIRA, 1984). O PD também mantém maiores taxas de infiltração de água por evitar a formação de crostas superficiais e por aumentar o tempo de oportunidade da infiltração, por causa da maior rugosidade da superfície. Além disso, os resíduos atuam na conservação da água pela redução das taxas de evaporação decorrentes da reflexão de energia radiante. Costa et al. (2003), após 21 anos de manejo em Guarapuava (PR), concluíram que o solo em PD apresentou maior umidade volumétrica do que o solo em PC na camada de $0-10 \mathrm{~cm}$. Na camada $10-20$ $\mathrm{cm}$, a umidade do solo não diferiu entre os sistemas de manejo. Dois fatores contribuíram para a maior umidade na camada superficial em PD: a maior infiltração de água, decorrente da não-formação de selamento superficial e a menor evaporação de água, decorrente da cobertura do solo por resíduos. Abreu, Reichert e Reinert (2004) encontraram que o solo descoberto apresentou a maior condutividade, sendo similar àquela encontrada para cultivo mínimo- crotalária e escarificação-soja. Na profundidade de $12 \mathrm{~cm}$, não se observou diferença significativa entre os tratamentos.

Souza e Alves (2003), ao avaliar seis sistemas de manejo no cerrado, mediram a condutividade hidráulica de cada sistema em três profundidades. $\mathrm{O}$ cerrado apresentou as maiores condutividades hidráulicas em todas as profundidades, diferindo estatisticamente dos demais sistemas de manejo. Os sistemas cultivo mínimo e PD mostraram valores intermediários e não diferiram estatisticamente. Os sistemas seringueira, pastagem e convencional, indicaram os menores valores, com destaque para o sistema da seringueira, menor dos três nas profundidades $0-10 \mathrm{~cm}$ e $10-20 \mathrm{~cm}$. Nos sistemas de cerrado e cultivo mínimo, a condutividade hidráulica do solo saturado diminui em profundidade e nos sistemas com PD, seringueira, pastagem e preparo convencional, não houve diferença entre as profundidades em relação à condutividade hidráulica. A maior condutividade hidráulica do solo saturado nos sistemas de PD e cultivo mínimo pode ser explicada pela continuidade dos poros e pela maior atividade biológica da micro, meso e macrofauna, sendo que este fato é concordante com os resultados obtidos por Arzeno (1990), Sarvasi (1994) e Castro (1995).

Oliveira et al. (2004), na avaliação de sistemas de manejo em Latossolo Vermelho distrófico típico sob cerrado, em Planaltina (DF), concluíram que a semeadura direta apresentou maior disponibilidade de água que o sistema arado de discos e cerrado na profundidade $0-5 \mathrm{~cm}$. Araújo et al. (2004b), ao estudar o efeito da escarificação após treze anos de semeadura direta em Latossolo Vermelho distroférrico de Campo Mourão (PR), concluíram que a escarificação do solo promoveu efeitos benéficos na camada $0-15 \mathrm{~cm}$, principalmente em relação à porosidade de aeração. No entanto, na camada 15$30 \mathrm{~cm}$, a escarificação reduziu o intervalo ótimo de tensão de água no solo (IOP), principalmente em virtude da resistência do solo à penetração que se comportou como limite superior em toda a faixa de variação do IOP e os resultados sugerem que, neste 
solo sob este manejo, não é necessária a escarificação.

A título de síntese, verificou-se que o preparo mínimo e o plantio direto apresentam maior taxa de infiltração e maior condutividade hidráulica comparados a outros sistemas de manejo e são mais sustentáveis no que diz respeito à água do solo.

\section{Compressibilidade do solo}

Compressibilidade do solo é a facilidade com que um solo não saturado decresce de volume quando sujeito a pressões, sendo variável em função do tipo, intensidade e freqüência de carga aplicada e da tensão da água no solo, umidade, textura, estrutura e densidade inicial (DIAS JÚNIOR; PIERCE, 1996).

No modelo desenvolvido por Dias Júnior e Pierce (1996), a pressão de preconsolidação representa o valor máximo da pressão que poderá ser aplicada ao solo para evitar compactação adicional. Tudo se resume a definir quando o solo está com a umidade adequada para as operações de mecanização e estimar quanta deformação ocorreria quando as pressões aplicadas pelas máquinas e equipamentos excedessem sua capacidade de suporte. A umidade adequada, então, é aquela que indicará a pressão máxima que o solo pode suportar sem compactação adicional (pressão de preconsolidação). A pressão de preconsolidação pode ser determinada das curvas de compressão do solo, obtidas em ensaios de compressão uniaxial (DIAS JUNIOR, 1994; SILVA; REINERT; REICHERT, 2000b; OLIVEIRA et al., 2003a; OLIVEIRA; DIAS JUNIOR; RESK, 2003b; SILVA et al., 2003a; SILVA et al., 2003b). Segundo Oliveira et al. (2003a), a estimativa é de que as pressões médias aplicadas aos solos pelas máquinas agrícolas se situem entre 50 e $300 \mathrm{KPa}$ (LEBERT; HORN, 1991; CARPENEDO, 1994; SILVA et al., 2000a). Os reboques e caminhões podem exercer pressões entre 300 e $600 \mathrm{KPa}$ (CARPENEDO, 1994; SILVA; REINERT; REICHERT, 2000a).

Oliveira et al. (2003a) e Oliveira et al. (2003b) utilizaram o modelo para as condições de cerrado, avaliando as alterações estruturais e o comportamento compressivo de um Latossolo Vermelho distrófico, em área do CPAC-EMBRAPA em Planaltina (DF) sob cerrado, PD e preparo com arado de discos (AD), após duas décadas de uso e manejo. Os resultados obtidos indicam que as tensões de água no solo afetam a pressão de preconsolidação tanto sob cerrado, como em PD e AD, sendo os valores mais elevados nas tensões mais baixas. $\mathrm{Na}$ profundidade $0-5 \mathrm{~cm}$, na tensão de água de 1.500 $\mathrm{kPa}$ (Ponto de Murcha Permanente-PMP), o cerrado $(314 \mathrm{kPa})$ e o PD (320 kPa) suportaram mais carga em relação à condição de preparo com arado de discos $(275 \mathrm{kPa})$. Na profundidade $20-30 \mathrm{~cm}$, o solo sob preparo com arado de discos $(326 \mathrm{kPa})$ suportou mais carga em relação aos demais sistemas. Para trabalho com máquinas na zona de friabilidade, constata-se que, na profundidade de $0-5 \mathrm{~cm}$, os valores de pressão a serem aplicados ao solo para evitar a compactação variaram de 144 a $321 \mathrm{kPa}$, para tensões de água entre -11 e $-2.010 \mathrm{kPa}$, sob cerrado; 180 a $258 \mathrm{kPa}$, para tensões de água no solo entre-16 e-188 kPa, sob PD; e 152 a $209 \mathrm{kPa}$, para tensões de água entre -12 e $-102 \mathrm{kPa}$, sob arado de discos. Na profundidade de $20-30 \mathrm{~cm}$, os valores de pressão que devem ser aplicados variaram de 138 a $314 \mathrm{kPa}$, para tensões de água entre -6 e $1.397 \mathrm{kPa}$, em solo sob Cerrado; 162 a $237 \mathrm{kPa}$, para tensões de água entre -10 e $-188 \mathrm{kPa}$, sob PD; e 193 a $274 \mathrm{kPa}$, para tensões de água entre $-8 \mathrm{e}-$ $175 \mathrm{kPa}$, sob preparo com arado de discos. Levando em conta os valores de pressão aplicada aos solos pelas máquinas agrícolas de 50 a $300 \mathrm{kPa}$, há necessidade de cautela na tomada de decisão do momento mais adequado para o uso de máquina no campo, pois mesmo estando na zona de friabilidade, pode ocorrer compactação do solo, pois a capacidade de suporte de carga do solo pode ser excedida.

Estudo feito por Silva et al. (2003a) avaliou o efeito do tráfego sob a ótica da pressão de preconsolidação, também em Latossolo Vermelho distrófico, em Planaltina (DF), onde foram preparadas parcelas com arado de discos, arado de aivecas, grade 
aradora e semeadora-adubadora (PD) e foram avaliadas as pressões de preconsolidação antes do preparo, logo após a passada do rodado traseiro no preparo e após a colheita. Concluiu-se que, na camada superficial, as maiores deformações ocorreram logo depois da passada do rodado traseiro. $\mathrm{O}$ arado de aivecas provocou um incremento na pressão de preconsolidação de $26 \%$, seguido do arado de discos com 25\%, da grade aradora com $20 \%$ e da semeadora/adubadora, com 17\%. Após a colheita houve uma redução da pressão de preconsolidação no arado de aivecas em 11\%, na grade aradora em $10 \%$ e no arado de discos em $2 \%$, enquanto que na semeadora/adubadora houve aumento de $0,8 \%$. A intensidade de tráfego do rodado e a ação da soleira dos implementos alteraram a compressibilidade, a densidade, a porosidade e a condutividade hidráulica do solo saturado nas profundidades superficial, média de trabalho e de corte dos implementos. De maneira geral, a passada do rodado traseiro aumentou os valores de pressão de preconsolidação do solo na superfície, enquanto os tráfegos subseqüentes, necessários ao cultivo, elevaram esses valores em subsuperfície, tanto na profundidade média de trabalho como na profundidade de corte sob o implemento.

Mesmo com o avanço teórico da modelagem das tensões de pré-consolidação é necessária sua validação para as múltiplas situações da agricultura brasileira, de forma a gerar mapas de trafegabilidade de fácil utilização, capazes de minimizar os impactos mais ameaçadores da compactação.

\section{Níveis de carbono orgânico e estabilidade de agregados}

Agregados de solo maiores e mais pesados conferem maior estruturação ao solo, maior porosidade, maior condutividade hidráulica e maior resistência à compressão com inegáveis vantagens para a sustentabilidade dos sistemas de produção. Segundo Castro Filho, Muzilli e Podanoschi (1998) o tamanho dos agregados do solo e o estado de agregação podem ser influenciados por diferentes processos de manejo e práticas culturais, que alteram o teor de matéria orgânica e a atividade biológica do solo. A matéria orgânica é considerada por muitos pesquisadores como o principal agente de estabilização dos agregados do solo (TISDALL; OADES, 1982; CASTRO FILHO; MUZILLI; PODANOSCHI, 1998; GANG LU et al., 1998) e segundo Cruz et al. (2003) o resultado esperado em tratamentos que apresentem diferenças no teor de carbono orgânico é que haja uma alta correlação entre carbono orgânico e agregação do solo. Além disso, o ambiente menos oxidativo no solo em PD, promove diminuição no grau de humificação da matéria orgânica em comparação aos solos manejados sob preparo reduzido e preparo convencional (BAYER; MARTINNETO; SAAB, 2003).

Castro Filho, Muzilli e Podanoschi (1998), após catorze anos de manejo de solo diferenciado em Latossolo Roxo em Londrina (PR), concluíram que o sistema de PD, pelo acúmulo de resíduos vegetais na superfície, melhorou o estado de agregação graças ao incremento de C-orgânico, sobretudo na camada $0-10 \mathrm{~cm}$, onde os valores de diâmetro médio geométrico (DMG) e diâmetro médio ponderado (DMP) dos agregados foram significativamente superiores no PD em relação ao PC. O aumento de C-orgânico resultou em aumento dos índices de agregação, pela diminuição da classe de menor diâmetro e aumento das classes de diâmetro maior. A agregação do solo tendeu a aumentar em rotações de culturas com espécies de relação $\mathrm{C} / \mathrm{N}$ mais alta, como o milho.

Após sete anos de experimentação em um Latossolo Bruno álico de Guarapuava (PR), na altitude de 1000m, Eltz, Peixoto e Jaster (1989) concluíram que não houve diferenças significativas entre sistemas de manejo no C-orgânico, mas na camada $0-5 \mathrm{~cm}$ o PD apresentou diâmetro médio de agregados significativamente superior $(5,5 \mathrm{~mm})$ ao PC (2,0 a 3,0 mm). Bertol et al. (2004), em Cambissolo Húmico, a $940 \mathrm{~m}$ de altitude em Lages (SC), após sete anos, concluíram que o teor de carbono orgânico foi maior no PD e no campo nativo 
do que no preparo convencional, especialmente na superfície do solo, diminuindo com a profundidade em todos os sistemas de manejo. Isso influenciou o diâmetro médio ponderado de agregados, que foi menor no preparo convencional $(4,7 \mathrm{~mm})$ do que no PD (5,9 mm) e no campo nativo $(6,0 \mathrm{~mm})$. Cruz et al. (2003), para um Argissolo Vermelho francoarenoso, em Pelotas (RS), encontraram maior conteúdo de carbono orgânico no $\mathrm{PD}$; mas isso não provocou diferenças no diâmetro médio de agregados, talvez devido ao fato do sistema PD ter sido estabelecido há apenas três anos, insuficientes para alterar a estrutura. Rosa et al. (2003), em cultivo intensivo em Latossolo Vermelho eutroférrico argiloso, em Santa Helena de Goiás (GO), após cinco anos de PD irrigado, PD de sequeiro e floresta natural (cerradão), concluíram que o PD não foi capaz de restabelecer a matéria orgânica nos níveis da mata, além de favorecer a compactação do solo estudado. O PD de sequeiro não acarretou variações significativas nos teores de carbono orgânico total e nas formas mais dinâmicas de carbono, quando comparado ao PD irrigado. Materechera e Mkhabela (2001) verificaram o efeito estruturante da matéria orgânica no sudeste da Swazilândia, comparando a camada $0-15 \mathrm{~cm}$ de um solo cultivado continuamente, que apresentou 1,08\% de C-orgânico e diâmetro médio geométrico úmido (DMG) de 1,6 $\mathrm{mm}$, um solo sob pousio que apresentou $1,83 \%$ de C-orgânico e DMG de 2,2 mm e um antigo criadouro usado para cultivo que apresentou 3,90\% de Corgânico e DMG de 2,9 mm. Kay e Vandenbygaart (2002) afirmam que as pesquisas têm indicado que o C-orgânico do solo se acumula perto da superfície e é perdido nas camadas subsuperficiais quando da conversão do PC para o PD. Há necessidade de mais pesquisas sobre como o balanço entre esses dois processos muda com o tempo em solos de diferentes texturas, drenagens e sob diferentes climas. Como exemplo de longo prazo, Castro Filho et al. (2002), trabalhando em Latossolo Vermelho distrófico após 21 anos de comparação entre PD e $\mathrm{PC}$, encontraram que o peso médio $(7,171$ e $5,845 \mathrm{~g}$ respectivamente), o diâmetro médio (4,266 e 2,844 mm respectivamente) e o índice de estabilidade de agregados (93 e 90 respectivamente) foram significativamente maiores em PD que em PC. Oliveira et al. (2003a), após 20 anos de manejo de um Latossolo Vermelho distrófico de cerrado em Planaltina (DF), encontraram diâmetro médio geométrico (DMG) de agregados maior em cerrado $(4,4 \mathrm{~mm})$ que em PD $(3,6 \mathrm{~mm})$ e convencional $(3,3 \mathrm{~mm})$ na camada $0-5 \mathrm{~cm}$, com matéria orgânica de 48,48 e $28 \mathrm{~g} \mathrm{~kg}^{-1}$ respectivamente. Já na profundidade $20-30 \mathrm{~cm}$ não houve diferença entre sistemas, com 4,3 mm de DMG e matéria orgânica de $29 \mathrm{~g} \mathrm{~kg}^{-1}$ para os três. Costa et al. (2003), em uma avaliação de vinte e um anos comparando PC, PD e mata nativa em Latossolo Bruno na altitude de $1000 \mathrm{~m}$, em Guarapuava (PR), concluíram que o maior DMG dos agregados foi observado na camada $0-5 \mathrm{~cm}$ sob mata $(5,2 \mathrm{~mm})$, seguido de PD $(3,7 \mathrm{~mm})$ e PC $(1,6 \mathrm{~mm})$, indicando efeito positivo do nãorevolvimento do solo e do acúmulo de resíduos vegetais na superfície sobre a estabilidade de agregados. Efeitos semelhantes do PD no aumento da estabilidade de agregados têm sido verificados em Argissolos (ALBUQUERQUE; REINERT; FIORIN, 1994) e Latossolos (CAMPOS et al., 1995) do sul do Brasil.

De forma geral, o sistema de plantio direto tem acumulado carbono orgânico e tem formado agregados maiores na superfície em relação ao plantio convencional, aproximando-se mais das condições de mata, cerrado e campo nativo, sendo mais sustentável com relação a esses aspectos.

\section{Considerações finais}

A densidade total, a porosidade e o tamanho de poros são indicadores necessários, mas não suficientes, para avaliar qualidade física do solo. $\mathrm{O}$ aumento da densidade superficial em plantio direto, de certa forma explica a atitude de parte dos agricultores em adotar a escarificação eventual. 
Solos sob preparo mínimo apresentam resistência à penetração semelhante aos solos sob cerrado, bastante inferior ao plantio direto e convencional, o que adiciona novos elementos à discussão da sustentabilidade.

O preparo mínimo e o plantio direto apresentam maiores taxas de infiltração e maiores condutividades hidráulicas quando comparados a outros sistemas de manejo convencional.

É necessário buscar formas de racionalizar o tráfego de máquinas e observar a umidade adequada para realização de operações, principalmente em plantio direto, que armazena mais umidade e cujo acúmulo de palha predispõe à patinagem. A determinação das pressões de pré-consolidação e pressões críticas para diferentes sistemas, tipos de solos e regiões pode determinar momentos ótimos de umidade para realização de operações mecanizadas, de forma a minimizar o incremento da compactação em direção a níveis críticos.

A matéria orgânica tem papel preponderante na estrutura do solo e na estabilidade de agregados, e é um dos fatores mais importantes na concessão de maior resistência à compactação em plantio direto e em preparo mínimo, capaz de garantir a sustentabilidade desses sistemas no longo prazo.

Os sistemas de plantio direto e de preparo mínimo se aproximam, em alguns aspectos, das condições naturais e apresentam mais aspectos favoráveis de sustentabilidade, mas não reproduzem exatamente as condições das coberturas naturais originais de mata, cerrado ou campos nativos.

\section{Referências}

ABRÃO, P. U. R.; GOEPFERT, C. F.; GUERRA, M.; ELTZ, F. L. F.; CASSOL, E. A. Efeitos de sistemas de preparo do solo sobre características de um Latossolo Roxo distrófico. Revista Brasileira de Ciências do Solo, Campinas, v.3, p.169-172, 1979.

ABREU, S. L.; REICHERT, J. M.; REINERT, D. J. Escarificação mecânica e biológica para a redução da compactação em Argissolo franco-arenoso sob plantio direto. Revista Brasileira de Ciências do Solo, Campinas, v.28, n.3, p.519-531, 2004.

ALBUQUERQUE, J. A.; REINERT, D. J.; FIORIN, J. E. Variação temporal da estabilidade estrutural em Podzólico Vermelho-Amarelo. Ciência Rural, Santa Maria, v.24, p.275-280, 1994.

ALLMARAS, R. R.; BLACK, A. L.; RICKMAN, R. W. Tillage, soil environment and root growth. In: CONSERVATION TILLAGE CONFERENCE, 1973, Des Moines. Proceedings... Des Moines, 1973. p.28-30.

ALVES SOBRINHO, T. Infiltração de água no solo em sistemas de plantio direto e convencional. Revista Brasileira de Engenharia Agricola e Ambiental, Campina Grande, v.7, n.2, p.191-196, 2003.

ANGERS, D. A.; BOLINDER, M. A .; CARTER, M. R.; GREGORICH,E.G.;DRURY,C.F.; LIANG,B.C.;VORONEY, R. P.; SIMARD, R. R.; DONALD, R. G.; BEYAERT, R. P. Impact of tillage practices on organic carbon and nitrogen storage in cool, humid soils of eastern Canada. Soil and Tillage Research, Amsterdam, v.41, p.191 - 201, 1997.

ARAÚJO, M. A.; TORMENA, C. A.; INOUE, T. T.; COSTA, A. C. S. Efeitos de uma escarificação na qualidade física de um Latossolo Vermelho distroférrico após treze anos de semeadura direta. Revista Brasileira de Ciências do Solo, Campinas, v.28, n.3, p.495-504, 2004 b.

ARAúJo, M. A.; TORMENA, C. A.; SILVA, A. P. Propriedades físicas de um Latossolo Vermelho distrófico cultivado e sob mata. Revista Brasileira de Ciências do Solo, Campinas, v.28, n.2, p.337-345, 2004a .

ARZENO, J. L. Avaliação física de diferentes manejos de solo em um Latossolo Roxo - distrófico. 1990. Tese (Doutorado) - Escola Superior de Agronomia Luiz de Queiroz/USP, Piracicaba.

AZOOZ, R. H., ARSHAD, M. A., FRANZLUEBBERS, A. J., Pore size distribution and hydraulic conductivity affected by tillage in northwestern Canada. Soil Science Society of America, Madison, v.60, n.1, p.1197-1201, 1996.

BAYER, C.; MARTIN-NETO, L.; SAAB, S. C. Diminuição da humificação da matéria orgânica de um cambissolo húmico em plantio direto. Revista Brasileira de Ciências do Solo, Campinas, v.27, n.3, p.537-544, 2003.

BELTRAME, L. F. S.; GONDIM, L. A. P.; TAYLOR, J. C. Estrutura e compactação na permeabilidade de solos do Rio Grande do Sul. Revista Brasileira de Ciências do Solo, Campinas, v.5, p.145-149, 1981.

BERTOL, I.; ALBUQUERQUE, J. A.; LEITE, D.; AMARAL, A. J.; ZOLDAN JÚNIOR, W. Propriedades físicas do solo sob preparo convencional e semeadura direta em rotação e sucessão de culturas, comparadas às do campo nativo. 
Revista Brasileira de Ciências do Solo, Campinas, v.28, n.1,p.155-163, 2004

BERTOL, I.; SCHICK, J.; MASSARIOL, J. M.; REIS, E. F.; DILY, L. Propriedades físicas de um Cambissolo Húmico álico afetadas pelo manejo do solo. Ciência Rural, Santa Maria, v.30, p.91-95, 2000.

BORGES, A. L.; KIEHL, J.; SOUZA, L. S. Alteração de propriedades físicas e atividade microbiana de um Latossolo Amarelo álico após cultivo com fruteiras perenes e mandioca. Revista Brasileira de Ciências do Solo, Campinas, v.23, p.1019-1025, 1999.

CAMPOS, R. C.; REINERT,D. J.; NICOLODI, R.; RUEDELL, J.; PETRERE, C. Estabilidade estrutural de um Latossolo Vermelho-Escuro distrófico após sete anos de rotação de culturas e sistemas de manejo do solo. Revista Brasileira de Ciências do Solo, Campinas, v.19, p.121-126, 1995.

CARPENEDO, V. Compressibilidade de solos em sistemas de manejo. 1994. Tese (Doutorado em Ciências do Solo)Universidade Federal do Rio Grande do Sul, Porto Alegre.

CASTRO FILHO, C.; MUZILLI, O.; PODANOSCHI, A. L. Estabilidade dos agregados e sua relação com o teor de carbono orgânico num Latossolo Roxo distrófico, em função de sistemas de plantio, rotações de culturas e métodos de preparo de amostras. Revista Brasileira de Ciências do Solo, Campinas, v.22, p.527-538, 1998.

CASTRO FILHO, C.; LOURENÇO, A.; GUIMARÃES, M. F.; FONSECA, I. C. B. Aggregate stability under different soil management systems in a red latosol in the state of Paraná, Brazil. Soil and Tillage Research, Amsterdam, v.65, p.45-51, 2002.

CASTRO, O. M. Comportamento fisico e químico de um latossolo Roxo em função do seu preparo na cultura do milho (Zea mays l.).1995. Tese (Doutorado) - Escola Superior de Agronomia Luiz de Queiroz, Piracicaba.

CORRECHEL, V.; SILVA, A. P.; TORMENA, C. A. Resistência de um Latossolo Roxo em dois sistemas de preparo do solo. In:CONGRESSOBRASILEIRODECIÊNCIADOSOLO,26., 1997, Rio de Janeiro. Anais... Rio de Janeiro: Sociedade Brasileira de Ciências do Solo,1997.p.20.

CORSINI, P. C.; FERRAUDO, A. S. Efeitos de sistemas de cultivo na densidade e na macroporosidade do solo e no desenvolvimento radicular do milho em Latossolo Roxo. Pesquisa Agropecuária Brasileira, Brasília, v.34, n.2, p.289-298, fev. 1999.

COSTA, F. S.; ALBUQUERQUE, J. A.; BAYER, C.; FONTOURA, S. M. V.; WOBETO, C. Propriedades físicas de um Latossolo Bruno afetadas pelos sistemas de plantio direto e preparo convencional. Revista Brasileira de Ciências do Solo, Campinas, v.27, n.3, p.527-535, 2003.
CRUZ, A. C. R.; PAULETTO, E. A.; FLORES, C. A.; SILVA, J. B. Atributos físicos e carbono orgânico de um argissolo vermelho sob sistemas de manejo. Revista Brasileira de Ciências do Solo, Campinas, v.27, n.6, p.1105-1112, 2003.

DALlA ROSA, A. Práticas mecânicas e culturais na recuperação de solos degradados pelo cultivo. 1981. Dissertação (Mestrado)- Faculdade de Agronomia/ Universidade Federal do Rio Grande do Sul, Porto Alegre.

DE MARIA, I. C.; CASTRO, O. M.; SOUZA DIAS, H. Atributos físicos do solo e crescimento radicular de soja em Latossolo Roxo sob diferentes métodos de preparo do solo. Revista Brasileira de Ciências do Solo, Campinas, v.23, p.703-709, 1999.

DENARDIN, J. E.; KOCHHANN, R. A. Pesquisa de desenvolvimento em sistema plantio direto no Rio Grande do Sul. In: CONGRESSO BRASILEIRO DE CIÊNCIA DO SOLO, 26., Rio de Janeiro, 1997. Anais... Rio de Janeiro, 1997. CD ROM

DERPSCH, R.; ROTH, C.; SIDIRAS, N.; KÖPKE, U. Controle de erosão no Paraná, Brasil: Sistemas de cobertura do solo, plantio direto e preparo conservacionista do solo. Eschborn: Deutsche Gesellschaff für Technische Zusammenarbeit, 1991.

DIAS JUNIOR, M. S. Compression of three soils under long-term tillage and wheel traffic. 1994. Thesis (Ph.D. in Crop and Soil Science) - Michigan State University, East Lansing, 1994.

DIAS JUNIOR, M. S.; PIERCE, F. J. O processo de compactação do solo e sua modelagem. Revista Brasileira de Ciências do Solo, Campinas, v.20, p.175-182, 1996.

DOUGLAS, J. T.; JARVIS, M. G.; HOWSE, K. R.; GOSS, M. J. Structure of a silty soil in relation to management. The Journal Of Soil Science, Oxford, v.37, p.137-151. 1986

EKEBERG, E., RILEY, H. C. F. Tillage intensity effects on soil properties and crop yields in a long-term trial on morainic loam soil in southeast Norway. Soil And Tillage Research, Amsterdam, v.42, p.277-293, 1997.

ELTZ, F. L. F.; PEIXOTO, R. T. G.; JASTER, F. Efeitos de sistemas de preparo de solo nas propriedades físicas e químicas de um Latossolo Bruno álico. Revista Brasileira de Ciências do Solo, Campinas, v.13, p.259-267, 1989.

FALLEIRO, R. M.; SOUZA, C. M.; SILVA, C. S. W.; SEDIYAMA, C. S.; SILVA, A. A.; FAGUNDES, J. L.. Influência dos sistemas de preparo nas propriedades químicas e físicas do solo. Revista Brasileira de Ciências do Solo, Campinas, v.27, n.6, p.1097-1104, 2003

FERNANDES, B.; GALLOWAY, H. M.; BRONSON, R. D. Efeito de três sistemas de preparo de solo na densidade aparente, na porosidade total e na distribuição dos poros 
em dois solos. Revista Brasileira de Ciências do Solo, Campinas, v.7, n.3, p.329-333, 1983.

FOLONI, J. S. S.; CALONEGO, J. C.; LIMA, S. L. Efeito da compactação no desenvolvimento aéreo e radicular de cultivares de milho. Pesquisa Agropecuária Brasileira, Brasília, v.38, n.8, p.947-953, 2003

FRANZLUEBBERS, A. J., ARSHAD, M. A., Soil organic matter pools during early adoption of conservation tillage in northwestern Canada. Soil Science Society of America, Madison., v.60, p.1422-1427, 1996.

GANG, L. U.; SAKAGAMI, K.; TANAKA, H.; HAMADA, $R$. Role of soil organic matter in stabilization of waterstable aggregates in soils under different types of land use. Soil Science And Plant Nutrition, Tokyo, v.44, p.147155, 1998.

GENRO JUNIOR, S. A. Alteração da compactação do solo com o uso de rotação de culturas no sistema plantio direto. 2002. Tese (Mestrado)- Universidade Federal de Santa Maria, Santa Maria.

GENRO JÚNIOR, S. A.; REINERT, D. J.; REICHERT, J. M. Variabilidade temporal da resistência à penetração de um Latossolo argiloso sob semeadura direta com rotação de culturas. Revista Brasileira de Ciências do Solo, Campinas, v.28, n.3, p.477-484, 2004.

GOMEZ, J. A.; GIRALDEZ, J. V.; PASTOR, M.; FERERES, E. Effects of tillage method on soil physical properties infiltration, and yield in an olive orchard. . Soil and Tillage Research, Amsterdam, v.52, p.167-175, 1999.

HAKANSSON, I.; VOORHEES, W. R.; RILEY, H. Vehiche and wheel factors influencing soil compaction and crop response in different traffic regimes. Soil and Tillage Research, Amsterdam, v.11, p.239-282, 1988.

HUSSEIN, I.; OLSEN, K. R.; SIEMENS, J. C. Long-term tillage effects on physical properties of eroded soil. Soil Science, Baltimore, v.163, p.970 - 981, 1998.

IMHOFF, S. C. Indicadores de qualidade estrutural e trafegabilidade de Latossolos e Argissolos Vermelhos. 2002. Tese (Doutorado)- Escola Superior Luiz de Queiroz/ USP, Piracicaba.

KAY,B.D.; VANDENBYGAART, A. J. Conservation tillage and depth stratification of porosity and soil organic matter. Soil and Tillage Research, Amsterdam., v.66, p.107-118, 2002.

KLEPKER, D.; ANGHINONI, I. Características físicas e químicas do solo afetadas por métodos de preparo e modos de adulação. Revista Brasileira de Ciências do Solo, Campinas, v.19, p.395-401, 1995.

LAL, R. No-tillage effects on soil properties and maize (Zea mays L.) production in Western Nigeria. Plant and Soil, Amsterdam, v.40, p.321-331, 1974.
LAL, R.; MAHUOBI, A. A.; FAUSSEY, P. G. Long-term tillage and rotation effects on properties of a central Ohio soil. Soil Science Society of America, Madison, v.58, p.517 $-522,1994$.

LEBERT, M.; HORN, R. A method to predict the mechanical strength of agricultural soils. Soil and Tillage Research, Amsterdam., v.19, p.275-286, 1991.

MACHADO, J. A.; BRUM, A. C. R. Efeito dos sistemas de cultivo em algumas propriedades físicas do solo. Revista Brasileira de Ciências do Solo, Campinas, v.2, p.81-84, 1978.

MAHBOUBI, A. A.; LAL, R.; FAUSSEY, N. R. Twentyeight years of tillage effects on two soils in Ohio. Soil Science Society of America, Madison, v.57, p.506 - 512, 1993.

MATERECHERA, S. A.; MKHABELA, T. S. Influence of land-use of a ferralitic soil under low external input farming in southeastern Swaziland. Soil and Tillage Research, Amsterdam., v.62, p.15-25, 2001.

OLIVEIRA, G. C.; DIAS JUNIOR, M. S.; RESK, D. V. S.; CURI, N. Alterações estruturais e comportamento compressivo de um Latossolo Vermelho distrófico argiloso sob diferentes sistemas de uso e manejo. Pesquisa Agropecuária Brasileira, Brasília, v.38, n.2, p.291-299, 2003a.

OLIVEIRA, G. C.; DIAS JUNIOR, M. S.; RESK, D. V. S.; CURI, N. Caracterização química e físico-hídrica de um Latossolo Vermelho após vinte anos de manejo e cultivo do solo. Revista Brasileira de Ciências do Solo, Campinas, v.28, n.2, p.327-336, 2004.

OLIVEIRA, G. C.; DIAS JUNIOR, M. S.; RESK, D. V. S.; CURI, N. Compressibilidade de um Latossolo Vermelho argiloso de acordo com a tensão de água no solo, uso e manejo. Revista Brasileira de Ciências do Solo, Campinas, v.27, n.5, p.773-781, 2003b.

RIBON, A. A.; TAVARES FILHO, J. Models for the estimation of the physical quality of a Yellow Red Latosol (Oxisol) under pasture. Brazilian Archives of Biology and Technology, Curitiba, v.47, n.1, p.25-31, 2004.

ROSA, M. E. C.; OLSZEVSKI, N.; MENDONÇA, E. S.; COSTA, L. M.; CORREIA, J. R. Formas de carbono em Latossolo Vermelho eutroférrico sob plantio direto no sistema biogeográfico do cerrado. Revista Brasileira de Ciências do Solo, Campinas, v.27, n.5, p.911-923, 2003.

SALTON, J. C.; MIELNICZUK, R. Relações entre sistemas de preparo, temperatura e umidade de um Podzólico Vermelho Escuro de Eldorado do Sul - RS. Revista Brasileira de Ciências do Solo, Campinas, v.19, n.2, 1995. 
SAMPAIO, G. V. Efeito de sistemas de preparo do solo sobre o consórcio milho-feijão (Phaseolus vulgaris L.) e sobre algumas propriedades fisicas e quimicas do solo. 1987. Tese (Mestrado) - Universidade Federal de Viçosa, Viçosa.

SARVASI, F. O. C. Dinâmica da água, erosão hídrica e produtividade das culturas em função do preparo do solo. 1994. Dissertação (Mestrado)- Escola Superior de Agricultura Luiz de Queiroz/ USP, Piracicaba.

SCHJONNING, P.; RASMUSSEN, K. J., Soil strength and soil pore characteristics for direct drilled and ploughed soils. Soil and Tillage Research, Amsterdam, v.57, p.6982,2000 .

SCHOENHOLTZ, S. H.; VAN MIEGROET, H.; BURGER, J. A. A review of chemical and physical properties as indicators of forest soil quality: challenges and opportunities. Forest Ecology and Management, Amsterdam, v.138, p.335-356, 2000.

SECCO, D. Estados de compactação de dois latossolos e suas implicações no comportamento mecânico e na produtividade de culturas. 2003. Tese (Doutorado) Universidade Federal de Santa Maria, Santa Maria.

SIDIRAS, N.; DERPSCH, R.; MONDARDO, A. Influência de diferentes sistemas de preparo do solo na variação da umidade e rendimento da soja, em Latossolo Roxo distrófico (Oxisol). Revista Brasileira de Ciências do Solo, Campinas, v.7, p.103-106, 1983.

SILVA, R. B.; DIAS JÚNIOR, M. S.; SANTOS, F. L.; FRANZ, C. A. B. Influência do preparo inicial sobre a estrutura do solo quando da adoção do sistema de plantio direto, avaliada por meio da pressão de preconsolidação. Revista Brasileira de Ciências do Solo, Campinas, v.27, n.6, p.961-971,2003b.

SILVA, R. B.; DIAS JÚNIOR, M. S.; SILVA, F. A. M.; FOLE, S. M. O tráfego de máquinas agrícolas e as propriedades físicas, hídricas e mecânicas de um Latossolo dos cerrados. Revista Brasileira de Ciências do Solo, Campinas, v.27, n.6, p.973-983, 2003a.

SILVA, V. R.; REINERT, D. J.; REICHERT, J. M. Susceptibilidade à compactação de um Latossolo Vermelho-Escuro e de um Podzólico Vermelho-Amarelo. Revista Brasileira de Ciências do Solo, Campinas, v.24, p.239-249, 2000a.

SILVA, V. R. Variabilidade espacial da resistência do solo à penetração, intervalo hídrico ótimo e parâmetros físico-hídricos influenciando a produção de culturas anuais. 2003. Tese (Doutorado) - Universidade Federal de Santa Maria, Santa Maria.

SILVA, V. R.; REINERT, D. J.; REICHERT, J. M. Resistência mecânica do solo à penetração influenciada pelo tráfego de uma colhedora em dois sistemas de manejo do solo. Ciência Rural, Santa Maria, v.30, n.5, p.795-801, 2000 b.
SINGER, M.; EWING, S. Soil quality. In: SUMNER, M. E. (Ed.) Handbook of soil science. Boca Raton: CRC Press, 2000. p.271-298

SIQUEIRA, N. S. Efeito de sistemas de preparo do solo sobre a cultura do feijão (Phaseolus vulgaris, L.) e sobre algumas propriedades físicas e químicas do solo. 1989.Tese (Doutorado) - Universidade Federal de Viçosa, Viçosa.

SIQUEIRA, N. S. Influência de sistemas de preparo sobre algumas propriedades quimicas e físicas do solo e sobre a cultura do milho (Zea mays L.). 1995. Tese (Doutorado) - Universidade Federal de Viçosa, Viçosa.

SOUZA, Z. M.; ALVES, M. C. Movimento de água e resistência do solo à penetração em um Latossolo Vermelho distrófico de cerrado, sob diferentes usos e manejos. Revista Brasileira de Engenharia Agrícola e Ambiental, Campina Grande, v.7, n.1, p.18-23, 2003.

STONE, L. F.; SILVEIRA, P. M. Efeitos do sistema de preparo e da rotação de culturas na porosidade e densidade do solo. Revista Brasileira de Ciências do Solo, Campinas, v.25, p.395-401, 2001.

STRECK, C. A. Compactação do solo e seus efeitos no desenvolvimento e produtividade do feijoeiro e da soja. 2003. Tese (Doutorado) - Universidade Federal de Santa Maria, Santa Maria.

TAVARES FILHO, J. M. C.; BARBOSA, G.; GUIMARÃES, M. F.; FONSECA, I. C B. Resistência à penetração e desenvolvimento do sistema radicular do milho (Zea mays) sob diferentes sistemas de manejo em Latossolo Roxo. Revista Brasileira de Ciência do Solo, Campinas, v.25, n.3, p.725-720, 2001.

TAYLOR, H. M.; GARDNER, H. R. Penetration of cotton seedlings taproots as influenced by bulk density, moisture and strength of soil. Soil Science, Baltimore, v.96, p.153156, 1963.

TEBRÜGGE, F.; DÜRING, R. A. Reducing tillage intensitya review of results from a long-term study in Germany. Soil and Tillage Research, Amsterdam., v.53, p.15-28, 1999.

TISDALL, J. M.; OADES, L. M. Organic matter and water stable aggregates in soils. The Journal of Soil Science, Oxford, v.33, p.141-163, 1982.

TOPP, G. C.; REYNOLDS, W. D.; COOK, F. J.; KIRBY, J. M.; CARTER, M. R. Physical attributes of soil quality. In: GREGORICH, E. G.; CARTER, M. R. (Ed.) Soil quality for crop production and ecosystem health. Amsterdam: Elsevier Science, 1997. p.21-58.

TORMENA, C. A.; BARBOSA, M. C.; COSTA, A. C. S.; GONÇALVES, C. A. Densidade, porosidade e resistência à penetração em Latossolo cultivado sob diferentes sistemas de preparo de solo. Scientia Agrícola, Piracicaba, v.59, n.4, p.795-801 2002. 
TORMENA, C. A.; SILVA, A. P.; LIBARDI, P. L. Caracterização e avaliação do intervalo hídrico ótimo de um Latossolo Roxo. Revista Brasileira de Ciência do Solo, Campinas, v.22, p.573-581, 1998.

TORMENA, C. A.; ROLOFF, G. Dinâmica da resistência à penetração de um solo sob plantio direto. Revista Brasileira de Ciência do Solo, Campinas, v.20, p.333 339, 1996.

VALLEJOS, M. F. J. Influência de sistemas de preparo do solo em algumas propriedades químicas e fisicas de um Podzólico Vermelho-Amarelo câmbico argiloso, e na cultura do trigo (Triticum aestivum L.). 1998. Dissertação (Mestrado) - Universidade Federal de Viçosa, Viçosa.

VANDENBYGAART, A. J.; PROTZ, R.; TOMLIN, A. D. Changes in pore structure in a no-till chronosequence of silt loam soils, southern Ontario. Canadian Journal of Soil Science, Ottawa, v.79, p.149-160, 1999.
VIEIRA, M. J. Propriedades físicas do solo. In: INSTITUTO AGRONÔMICO DO PARANÁ. Plantio direto no estado do Paraná. Londrina: IAPAR, 1981. (Circular n.23).p.19-32.

VIEIRA, M. J.; COGO, N. P.; CASSOL; E. A. Perdas por erosão em diferentes preparos de solo para a cultura de soja em condições de chuva simulada. Revista Brasileira de Ciência do Solo, Campinas, v.2, n.3, p.209-214, 1978.

VIEIRA, M. J. O preparo do solo e o comportamento da planta. Plantio Direto, Ponta Grossa, v.1, n.5, p. 4-5, 1984.

WANG, D.; YATES, S. R.; ERNST, F. F. Determining soil hydraulic properties using tension infiltrometers, time domain reflectometry, and tensiometers. Soil Science Society of America, Madison, v.62, n.2, p.318-325, 1998.

YANG, X. M.; KAY, B. D. Impacts of tillage practices on total, loose- and occluded-particulate, and humified organic carbon fractions in soils within a field in southern Ontario. Canadian Journal Of Soil Science, Ottawa, v.81, p.149-156, 2001. 